\title{
Research of the Characteristics and Superiority of External Stimulation Oscillation Jet
}

\author{
Jiao Cheng ${ }^{1, a}$, Jiupeng Zou ${ }^{2, b}$, Weihua $X u^{3, c}$ and Peiqi Liu ${ }^{4, d}$ \\ ${ }^{1,2,3,4}$ School of Chemical Machinery, Dalian University of Technology, Ganjingzi District, Dalian, \\ Liaoning 116023, PR China \\ achengjiaodut@163.com, boujp@dlut.edu.cn, cweihuaxu361@163.com, 'Ipq21cn@dlut.edu.cn
}

\begin{abstract}
Keywords: Jet oscillation; Stimulation; Efficiency; Total pressure retention rate
Abstract. A novel external stimulation oscillator was recommended and its characteristics of high efficiency and easy oscillation were proved by CFD simulation and experiment. The superiority and difference of process mechanism of the oscillator compared with self-stimulation oscillator were analyzed. The geometric parameters of the new oscillator that could trigger and maintain efficient oscillation and the minimum stimulating flow were determined. It was also indicated that the redundant quantity of the stimulating flow would not decrease the total pressure retention rate as the energy of stimulating flow could be merged into the main jet.
\end{abstract}

\section{Introduction}

The oscillating jet has been used in flow measurement [1], boundary layer separation control [2], wing boundary layer amelioration to increase the lift [3] and so on. The static gas wave refrigerator relies on the periodic deflection of jet to distribute the jet into sector-arranged gas wave tubes [4]. In this way, the operational reliability problems have been solved such as shut-down by impurities or ice blocks and poor lubrication in low temperature, these appear in rotary shock wave refrigerator, heat separator and wave rotor expansion refrigerator. However, the significant obstacle of the generalization of static gas wave refrigerator is the low refrigeration efficiency. At present, the method of oscillating the jet is the distributary self-stimulation based on Coanda effect [5]. It is convenient but the loss of total pressure is over $30 \%$ which is one of the main factors that reduces the efficiency.

For the distributary of the self-stimulation, the kinetic energy and pressure are reduced as through the long channel, so the wider channel is needed to circulate enough incentive flow. And local vortex disturbs the high speed flow of the main jet. The energy decrease of the stimulating jet not only reduces the motivating ability, but also consumes the kinetic energy of the main jet obviously, for accelerating and carrying the stimulating flow as that of ejector.

A new stimulation manner is adopted to generate oscillation jet flow. It is a little stimulating flow imported from outside instead of the distributary of main jet. The pressure of the external flow equals to that of the main jet, so the flow could be accelerated spontaneously. The demanded stimulating flow is reduced significantly, and local vortex could also be weakened because of the smaller stimulating flow entrances. These would increase the efficiency of the oscillating process effectively.

Vertical and parallel stimulating flow work simultaneously to obtain better stimulation effect. This novel manner is named push-and-pull external stimulation [6].

\section{The Numerical Simulation of the Oscillation}

Numerical Model. Fig. 1(a) shows the simple structure of the external stimulation oscillator and Fig. 1(b) shows the structure of the positive feedback self-stimulation oscillator [7]. The total pressure of the nozzle inlet $P i=0.36 \mathrm{MPa}$, the pressure of the outlet $P o=0.18 \mathrm{MPa}$. The peak value of the total pressure of stimulating flow was $0.36 \mathrm{MPa}$, and the valley value was $0.18 \mathrm{MPa}$. The waveform of the stimulating flow was trapezoidal wave and the frequency was $50 \mathrm{~Hz}$.

The simulation was divided into two parts to explore the different mechanisms of the vertical and parallel stimulation. The computational grids are shown in Fig. 2(a) and 2(b). 


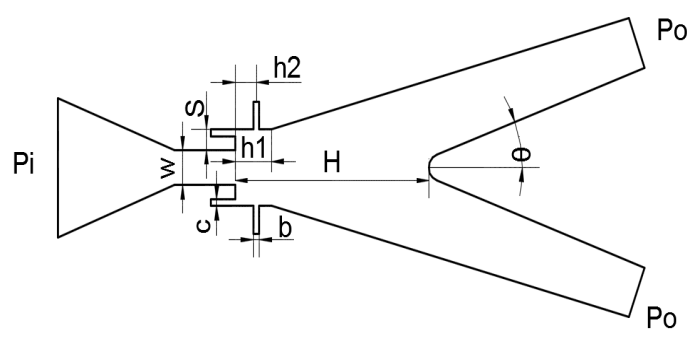

(a)

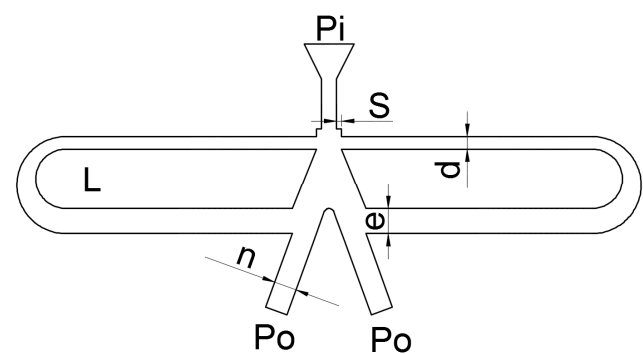

(b)

$W$ - the width of nozzle exit; $H$ - the distance between the nozzle exit and the spliter; $\theta$ - the angle of spliter; $h_{1}$ - straight length; $b, c$ - the width of the vertical and parallel stimulation orifice; $S$ - the position difference; $h_{2}$ - the distance between the nozzle exit and the vertical stimulation orifice; $e$ - the width of the distributary orifice; $d$ - the width of the feedback orifice; L- the length of control tube; $\mathrm{n}$ - the width of outlet.

Fig. 1 Structures of the two oscillators

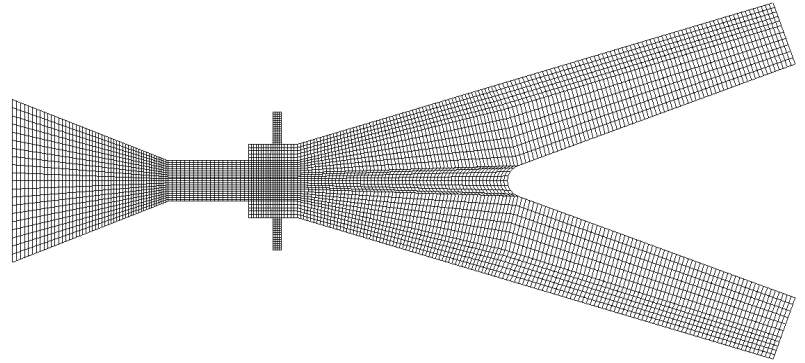

(a) The computational grid of vertical stimulation

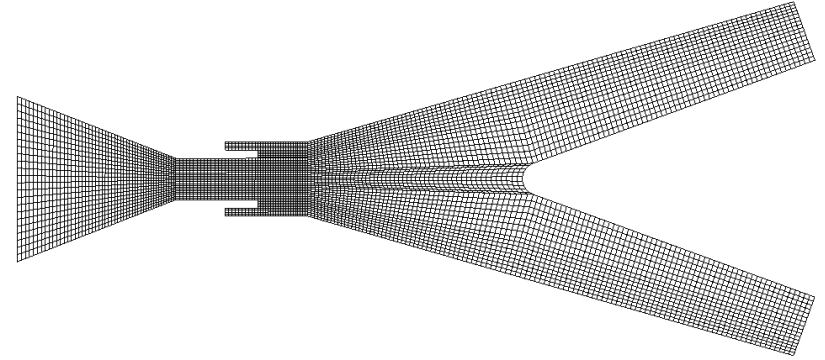

(b) The computational grid of parallel stimulation Fig. 2 Computational grids of the two simulations

The computational structured meshes were generated by ICEM. The FVM (finite volume method) was used to discrete the governing equations, the Roe flux difference splitting scheme was adopted for the convection term and the central difference scheme for the dissipation term. Second order implicit time step was chosen for unsteady integrals.

The Characteristic Dimensions and the Total Pressure Retention Rate. Two aspects were mainly concerned in numerical simulation, one was to investigate the effective characteristic sizes, another was to research the energy loss value in oscillating conditions. The energy loss value could be defined as total pressure retention rate $K$. It was the ratio of the mass-weighted total pressure of the outlet to the total pressure of the nozzle inlet. The higher the $K$, the lower the loss.

The computational dimensions were as follows: $W=2.5 \mathrm{~mm}, H=16 \mathrm{~mm}, \theta=20^{\circ}$ and for vertical stimulation $h_{1}=3 \mathrm{~mm}$, others were the variables. The results of numerical simulation are listed in Table 1 and Table 2.

Table 1 The simulation results of vertical stimulation oscillation

\begin{tabular}{cccccccc}
\hline$b[\mathrm{~mm}]$ & $S[\mathrm{~mm}]$ & $h_{2}[\mathrm{~mm}]$ & $K$ & $b[\mathrm{~mm}]$ & $S[\mathrm{~mm}]$ & $h_{2}[\mathrm{~mm}]$ & $K$ \\
\hline 0.20 & 0.50 & 1.75 & $84.53 \%$ & 0.5 & 1.25 & 1.75 & $83.32 \%$ \\
0.50 & 1.00 & 1.75 & $84.91 \%$ & 0.5 & 1.50 & 1.75 & $-{ }^{\mathrm{a}}$ \\
0.75 & 1.00 & 1.75 & $85.89 \%$ & 0.3 & 1.00 & 2.40 & $82.54 \%$ \\
0.50 & 0.75 & 1.75 & $84.25 \%$ & 0.3 & 1.00 & 2.85 & $82.92 \%$ \\
\hline
\end{tabular}

${ }^{\mathrm{a}}$ The main jet failed to oscillate.

Table 2 The simulation results of parallel stimulation oscillation

\begin{tabular}{cccccccc}
\hline$c[\mathrm{~mm}]$ & $S[\mathrm{~mm}]$ & $h_{1}[\mathrm{~mm}]$ & $K$ & $c[\mathrm{~mm}]$ & $S[\mathrm{~mm}]$ & $h_{1}[\mathrm{~mm}]$ & $K$ \\
\hline 0.15 & 0.75 & 3.00 & $79.49 \%$ & 0.50 & 1.50 & 3.00 & $78.38 \%$ \\
0.50 & 0.75 & 3.00 & $83.02 \%$ & 0.50 & 1.00 & 2.00 & $83.01 \%$ \\
0.50 & 1.25 & 3.00 & $81.42 \%$ & 0.50 & 1.00 & 5.00 & $82.52 \%$ \\
\hline
\end{tabular}




\section{Results Analysis and Experimental Verification}

Results Analysis. It is indicated that the oscillator could work steadily as $b, S$ and $h_{2}$ in a reasonable range. The characteristic parameters influence the total pressure retention rate $K$ obviously as well as the oscillation performance.

Under the optimized geometric conditions, the minimum value of $b / W$ that could sustain the oscillation is 0.08 , much better than the self-stimulation which requires $e=1.5 \mathrm{~W}$ and $d=W$.

The parallel stimulation alone is also able to trigger and sustain the oscillation, and the minimum value of $c / W$ is about 0.06 . While the push-and-pull stimulation could sustain the oscillation more stably and the total pressure retention rate $K$ could be increased. The different mechanisms between vertical and parallel stimulation will be discussed in another paper in detail.

The total pressure retention rate $K$ rises slightly with the increase of stimulating flow. The reason is that the static pressure of the stimulating inlet is higher than that of the downstream (see Fig. 3), the stimulating flow could be accelerated by self-expansion. So it scarcely consumes the energy of the main jet. Another reason is that the increment of the flow decreases the vortex loss in the branch channel. Therefore, the energy of the stimulating flow is not only applied to generate oscillation, it could also be superimposed to main jet and then be exploited. The total and static pressure nephogram at stimulation moments is shown in Fig. 3(a) and (b).

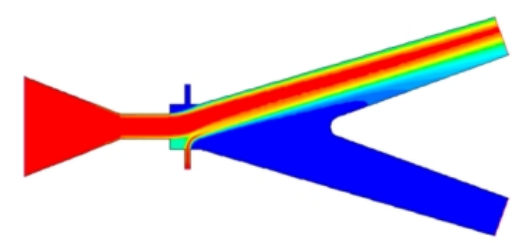

(a) Total pressure

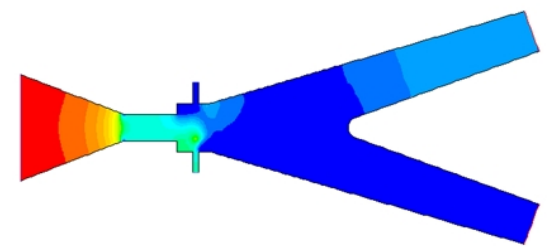

(b) Static pressure

Fig. 3 The total and static pressure nephogram of the vertical stimulation oscillation

The total pressure retention rate $K$ is over $80 \%$ in vertical or parallel stimulation. While it is over $90 \%$ in push-and-pull stimulation and the value is much more than that of self-stimulation as shown in Table 3.

Table 3 The simulation results of self-stimulation and push-and-pull stimulation oscillation

\begin{tabular}{cccccccc}
\hline \multicolumn{3}{c}{ self-stimulation oscillation } & \multicolumn{3}{c}{ push-and-pull stimulation oscillation } \\
$L[\mathrm{~mm}]$ & $n[\mathrm{~mm}]$ & $d[\mathrm{~mm}]$ & $K$ & $b[\mathrm{~mm}]$ & $c[\mathrm{~mm}]$ & $S[\mathrm{~mm}]$ & $K$ \\
\hline 73 & 4.5 & 5.0 & $68.0 \%$ & 0.50 & 0.50 & 1.50 & $84.89 \%$ \\
125 & 4.5 & 5.0 & $68.4 \%$ & 0.75 & 0.75 & 1.50 & $90.17 \%$ \\
125 & 5.0 & 5.0 & $65.8 \%$ & 0.75 & 0.75 & 2.00 & $90.70 \%$ \\
\hline
\end{tabular}

Experimental Verification. The experimental flowchart is shown in Fig. 4. The stimulating flow is modulated by reciprocating switching valve and the tested pressure waveform is shown in Fig. 5 (a). The actual pressure waveform of the outlet of oscillator is shown in Fig. 5 (b). The vibration exciter works under the control of the CS(control system).

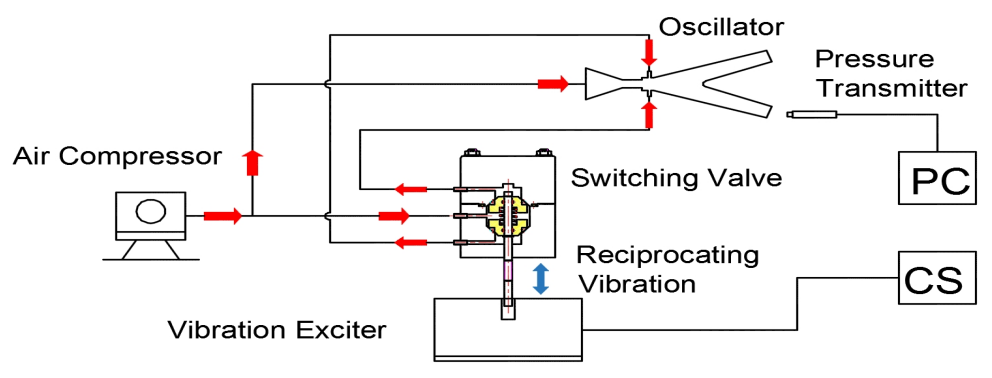

Fig. 4 The experimental flowchart 


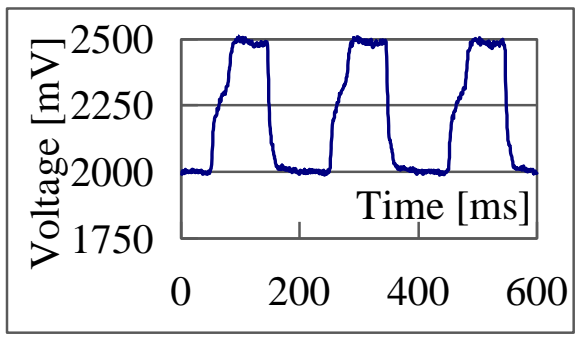

(a)

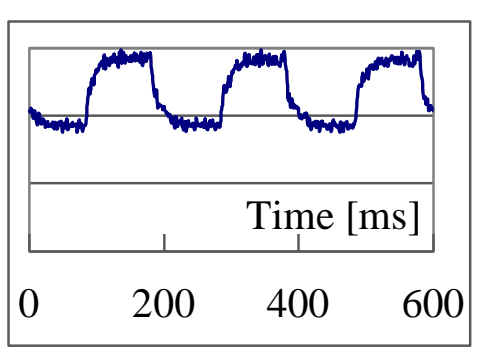

(b)

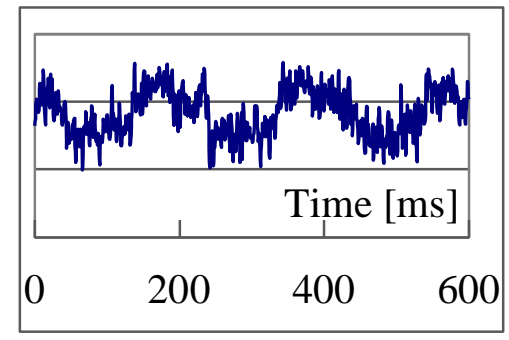

(c)

Fig. 5 The actual pressure waveform of the stimulating flow and the outlet

It has been proved that the oscillator could work steadily if $b \geq 0.08 \mathrm{~W}$. In reverse, the valley value of the waveform increased, indicating that the main jet failed to oscillate as shown in Fig. 5 (c).

The oscillator could generate a stable oscillation if the optimized sizes by simulation were adopted. It was demonstrated that the experimental results agreed well with the simulation results. Furthermore, the excellent follow performance of the main jet has been confirmed by experiment.

\section{Conclusions}

It is indicated by the simulation and experiment that the external micro jet stimulation oscillator is easier to oscillate, the efficiency is higher than that of the distributary self-stimulation oscillator by nearly $20 \%$, and the energy loss rate is reduced by almost $60 \%$ relatively.

The total pressure retention rate $K$ is over $82 \%$, and it is influenced significantly by geometric sizes especially the position difference $S$. Optimizing the geometric sizes, $K$ could be increased to over $90 \%$.

Under the conditions of optimized geometric sizes, the oscillation can be triggered and sustained if the vertical stimulating flow is over $8 \%$ of the main jet flow. The available value of $c / W$ is less than 0.08 . In particular, the push-and-pull stimulation can sustain the oscillation more stably.

A moderate increase of the stimulating flow would not decrease the total pressure retention rate and be more favorable to the oscillation. The energy of stimulating flow can merge into the main jet and be exploited. The optimized geometric sizes that may increase the total pressure retention rate should be preferred.

\section{Acknowledgements}

This work was supported by grants from the National Natural Science Foundation of China (51276026).

\section{References}

[1] Y.L. Bai and X. Ming: Chinese Journal of Scientific Instrument Vol. 29 No.4 (2008), p. 125 (In Chinese)

[2] C. Cerretelli and K. Kirtley: Journal of Turbomachinery Vol. 131 (2009), p. 041001-1

[3] J. Tang, Y.H. Li and F.P. Huo: Journal of Engineering Thermophysics Vol. 25 No.5 (2004), p. 765 (In Chinese)

[4] W. Liu and X.H. Ji: Journal of Refrigeration Vol. 30 No.3 (2009), p. 46 (In Chinese)

[5] S. Matsuo, T. Setoguchi and T. Kudo: Journal of Thermal Science Vol. 7 No.3 (1998), p.165

[6] J.P. Zou, D.P. Hu, Y.Q. Dai, C. Zhu, P.Q. Liu and L.M. Zhang, CHN. Patent 201410087495.6 (2014) (In Chinese)

[7] Y.R. LI, S. Someya, T. Koso, S. Aramaki and K. Okamoto: Flow Measurement and Instrumentation Vol. 33 (2013), p. 179 